\title{
THE EFFECT OF DIFFERENT COMBINATIONS OF CALCIUM HYDROXIDE FOR MANAGEMENT OF POST-OPERATIVE PAIN IN ACUTE APICAL PERIODONTITIS
}

\author{
Ajmal Yousaf, Fatima Ali, Afshan Bibi, Ahsan Masood Ahmed*, Sana Ashfaq, Syeda Fatima Tu Zahra \\ Armed Forces Institute of Dentistry/National University of Medical Sciences (NUMS) Rawalpindi Pakistan, *Medical Directorate, General Headquarter \\ Rawalpindi Pakistan
}

\begin{abstract}
Objective: To find the effect of different combinations of Calcium Hydroxide for the management of post-operative pain in acute apical periodontitis.

Study Design: Comparative cross-sectional study.

Place and Duration of Study: Department of Operative Dentistry, Armed Forces Institute of Dentistry, Rawalpindi Pakistan, from Jun to Nov 2019.

Methodology: A total of 282 patients presenting with acute apical periodontitis in their mandibular posterior teeth were selected for this study and were randomly divided into three equal groups with the help of scientific random number table. Group-A patients were given intracanal dressing of Calcium Hydroxide mixed with Chlorhexidine, group-B patients were given intracanal dressing of Calcium Hydroxide mixed with Saline and group-C patients were given intracanal dressing of Calcium Hydroxide mixed with Dexamethasone. Endodontic therapy was initiated after application of rubber dam, following pulpectomy and disinfection with frequent irrigation, canals were prepared and intra-canal dressings were placed according to the allotted group and the cavity was restored till the next appointment. Post-operative pain was recorded at 24 hours, 48 hours and one week using the visual analogue scale.

Results: The three intra-canal combinations of Calcium Hydroxide used in the study were found to be equally effective in reducing pain. Overall, the result between the three groups after 24 hours proved to be insignificant $(p=0.40)$, after 48 hours $(p=0.84)$ and 1 week $(p=0.45)$ were also insignificant.

Conclusion: Calcium Hydroxide mixed with Saline, Chlorhexidine and Dexamethasone are all effective for pain reduction with dexamethasone being the most effective.
\end{abstract}

Keywords: Apical periodontitis, Calcium hydroxide, Chlorhexidine, Dexamethasone, Post-operative pain.

How to Cite This Article: Yousaf A, Ali F, Bibi A, Ahmed AM, Ashfaq S, Zahra SFT. The Effect of Different Combinations of Calcium Hydroxide for Management of Post-Operative Pain in Acute Apical Periodontitis. Pak Armed Forces Med J 2021; 71(6): 2184-2188. Doi: https://doi.org/10.51253/pafmj.v71i6.3823

This is an Open Access article distributed under the terms of the Creative Commons Attribution License (https://creativecommons.org/licenses/by-nc/4.0/), which permits unrestricted use, distribution, and reproduction in any medium, provided the original work is properly cited.

\section{INTRODUCTION}

Endodontic treatment is often associated with mild to moderate pain and is usually linked to inflammatory reactions that may last from a few hours to several days. It may occur before, during or after endodontic therapy. The incidence of post-operative pain of low intensity in $2.53-58 \%$ of the cases is a common event even following all the standard protocols of endodontic therapy. ${ }^{1}$ Post-operative pain should be effectively managed to relive the patient's symptoms and to build a sense of trust between the patient and his clinician.

Various intracanal medicaments are used in dentistry. ${ }^{2}$ Teeth with necrotic pulps usually require multiple sessions for complete debridement and disinfection of the root canals and as a result between successive appointments, an intracanal dressing is placed for approximately one to two weeks for the above

Correspondence: Dr Fatima Ali, House No. 408, Street No. 13, Sector $\mathrm{B}$, Askari 14 Islamabad-Pakistan

Received: 28 Jan 2020; revision received: 11 May 2020; accepted: 12 May 2020 mentioned purposes. ${ }^{1}$ Calcium Hydroxide is one the most commonly used intracanal medicaments in patients undergoing multiple visit endodontic therapy and is quite effective because of its alkaline $\mathrm{pH}$ and antimicrobial properties promoting bacterial disinfection and healing. It is reported to be successful against a large number of bacterial species which causes endodontic failure. ${ }^{2}$ It may be used in combination with other agents to enhance the efficacy of its properties. Different vehicles can be used for its delivery into the root canals including distilled water, normal saline, chlorhexidine and different corticosteroid solutions. ${ }^{3,4} \mathrm{Sev}-$ eral studies have reported using Calcium Hydroxide in combination with Chlorhexidine as an intracanal dressing for post treatment pain. Chlorhexidine is an antibacterial that is effective against many gram positive and negative bacteria. ${ }^{5}$

A study was conducted by Midhat et al comparing the efficacy of Calcium Hydroxide alone and in combination with Chlorhexidine on pain reduction concluded that Calcium Hydroxide in combination 
with chlorhexidine resulted in reduced post treatment pain.

Corticosteroids are potent anti-inflammatory drugs that can be used either locally which is within the root canals as an intra-canal dressing or in systemic forms to reduce post-operative inflammation and promote healing. Little local literature is available comparing the effects of different combinations of Calcium Hydroxide on post-operative pain. The purpose of this study was to compare the effect of Calcium Hydroxide mixed with either chlorhexidine or saline or dexamethasone on post-operative pain in cases of acute apical periodontitis with irreversible pulpitis or necrotic pulp. The results of our study may be used as a reference and for comparison for further studies and research.

\section{METHODOLOGY}

After taking approval from the ethical committee (No 905/Trg-ABP1K2) of our institution, comparative cross-sectional study was carried out at Operative Department in Armed Forces Institute of Dentistry, Rawalpindi from June to November 2019. A total of 282 patients from 20-50 years of age from both gender with good general, mental and physical health were selected for this study and were randomly divided into three equal groups with the help of scientific random number table.

The sample size was calculated by using the WHO calculator, with a level of significance $5 \%$, power of test $80 \%$, population proportion $A=5.7 \%,{ }^{8}$ population proportion $\mathrm{B}=17.2 \%,{ }^{8}$ a total sample size of 282 patients was calculated with 94 patients in each of the three groups based on the following population proportions.

Inclusion Criteria: The mandibular posterior teeth with acute apical periodontitis with either irreversible pulpitis or necrotic pulps, without any intra-oral or extra-oral swelling or any draining sinus tract were selected for this study.

Exclusion Criteria: Patients on preoperative analgesics and antibiotics, teeth with calcified canals, open apex, previously traumatized teeth and root canal treated teeth along with immunocompromised patients, pregnant and lactating mothers were all excluded from the study.

The patients reporting to the Operative Dentistry Department of Armed Forces Institute of Dentistry, Rawalpindi requiring root canals of their mandibular posterior teeth were screened for inclusion by taking history, performing relevant clinical examination and necessary investigations along with peri-apical radiographs. The whole procedure of the study was explained to the patients in Urdu. After taking written informed consent from the willing participants of this study, the procedure was started.

Root canal treatment was initiated under local anesthesia and rubber dam isolation. Working length was established $1 \mathrm{~mm}$ from radiographic apex and was confirmed through peri-apical radiographs. The apical portion of canal was enlarged using K-files to size 3-4 files larger than the initial apical file, and the rest of the canal was prepared using the step-back technique of canal preparation. The canals were irrigated copiously with $2.5 \%$ Sodium hypochlorite in between the successive files to remove the necrotic pulp and the final irrigation was done using normal saline. Following instrumentation and irrigation, canals were dried with paper points of appropriate size and the intracanal medicament was placed in the canals in the following manner according to the groups allotted.

Group-A patients were dressed with Calcium Hydroxide mixed with 2\% Chlorhexidine. Group-B patients were dressed with Calcium Hydroxide mixed with saline. Group-C patients were dressed with Calcium Hydroxide mixed with Dexamethasone Phosphate $4 \mathrm{mg} / 1 \mathrm{ml}$.

Intracanal medicaments were inserted into the dried canals by means of a file that was at least two sizes smaller than the last file used to approximately 2 $\mathrm{mm}$ short from the apex in counterclockwise motion Excess material was removed from the pulp chamber and a sterile cotton pellet was placed over the opening of the root canal orifices and the teeth were sealed temporarily with cavit (zinc oxide eugenol based temporary filling material). At the end of every appointment patient was given a visual analogue scale (VAS), which was explained to the patient and the patient was advised to mark at the scale according to his pain intensity after 24 hours, 48 hours and one week of endodontic treatment and the patient was asked to bring these reading with him on the next scheduled visit. VAS is a $10 \mathrm{~cm}$ horizontal line with zero at one end which denotes 'no pain' and 10 on the other end which denotes 'unbearable pain'. This scale was used as a means for patient to record either the presence or absence of pain and the appointment for obturation was scheduled at 2 weeks after the initial visit.

Data was entered and analyzed using Statistical Package for the social sciences (SPSS) version 22.00. Frequencies and percentages were presented for 
qualitative variable like post-operative pain. Mean and standard deviation were calculated for qualitative variable like post-operative pain and for quantitative variables like age and gender; mean and standard deviation along with percentages in each group were calculated. Chi-square test was to compare the frequency of pain between the three groups of intra-canal medicaments after 24 hours, 48 hours and one week of initiation of endodontic treatment. The $p$-value of $\leq 0.05$ was considered significant. VAS scores of less than three $(<3)$ were considered as absence of pain and more than three $(>3)$ as presence of pain.

\section{RESULTS}

Out of total 282 patients, $48(51 \%)$ patient from Dexamethasone group, 45 (47.8\%) patients from Saline group and $40(42.5 \%)$ patients from Chlorhexidine groups, had no pain. The three intra-canal combinations of Calcium Hydroxide used in the study were found to be equally effective in reducing pain. Mean age of group-A was $33.43 \pm 5.60$ years, group-B was $31.93 \pm 4.70$ years and of group- $C$ was $31.35 \pm 6.7$ years. (Table-I).

Table-I: Age and gender distribution in Group-A, Group-B and Group-C.

\begin{tabular}{l|c|c|c}
\hline Variables & $\begin{array}{c}\text { Group A } \\
(\mathbf{n = 9 4 )}\end{array}$ & $\begin{array}{c}\text { Group B } \\
(\mathbf{n = 9 4 )}\end{array}$ & $\begin{array}{c}\text { Group C } \\
(\mathbf{n = 9 4 )}\end{array}$ \\
\hline Gender & $\mathbf{n ~ ( \% )}$ & $\mathbf{n ~ ( \% )}$ & $\mathbf{n ~ ( \% )}$ \\
\hline Male & $34(36.17)$ & $27(28.10)$ & $35(37.20)$ \\
\hline Female & $60(63.80)$ & $67(71.27)$ & $59(62.70)$ \\
\hline \multicolumn{3}{|l}{ Age (Years) } \\
\hline 21-30 & $25(26.60)$ & $40(42.60)$ & $35(37.20)$ \\
\hline $31-40$ & $33(35.10)$ & $28(29.80)$ & $42(44.70)$ \\
\hline $41-50$ & $36(38.30)$ & $26(27.70)$ & $17(18.10)$ \\
\hline
\end{tabular}

Our study found Calcium Hydroxide combination with Dexamethasone to be most effective for the first three days but after one week the difference between the three groups in pain reduction was not found to be statistically significant. All three combinations of Calcium Hydroxide effectively reduced the pain with Dexamethasone in combination with Calcium Hydroxide being the most effective. Overall, the result between the three groups after 24 hours proved to be insignificant $(p=0.40)$, after 48 hours $(p=0.84)$ and 1 week $(p=0.45)$ were also insignificant as shown in Table-II.

\section{DISCUSSION}

Pain after initiation of endodontic treatment is frequently encountered when the peri-radiclar tissues receive insult because of increased intensity of injury leading to acute exacerbations of underlying peri-radicular pathology. The incidence of post-operative pain has been found to be low in patients with a vital healthy pulp without any previous peri-radicular pathosis and is highest in patients who present with severe preoperative pain and swelling particularly with a necrotic diseased pulp and peri-radicular pathosis. 8 Several treatment approaches have been used to effectively reduce this incidence of pain; by giving pre-treatment analgesics, antibiotics, corticosteroids, placing intracanal dressings between appointments ${ }^{9}$, intra-canal irrigants, occlusal reduction and using low level laser, ${ }^{10}$ to name a few.

Calcium Hydroxide is one of the most commonly used and readily available intracanal medicament and it used either alone or in combination with various other intra-canal medicaments for enhancement of its anti-bacterial properties. It is normally used in the form of slurry formed by dissolving it in a water base. It dissolves readily in aqueous solution so water is commonly used as a vehicle but other solutions like saline and chlorhexidine are also used. In our study, Calcium Hydroxide was mixed with Saline, Chlorohexidine and Dexamethasone to check the effect on postoperative pain in cases of acute peri-apical periodontitis.

Table-II: Comparison of frequency of post-operative pain between group-A, B \& C after 24 hours, 48 hours and 1 week.

\begin{tabular}{|c|c|c|c|c|}
\hline Pain & $\begin{array}{l}\text { Group-A } \\
\text { (n=94) } \\
\text { Chlorhe- } \\
\text { xidine }\end{array}$ & $\begin{array}{c}\text { Group-B } \\
(\mathrm{n}=94) \\
\text { Saline }\end{array}$ & $\begin{array}{c}\text { Group-C } \\
(\mathrm{n}=94) \\
\text { Dexame- } \\
\text { thasone }\end{array}$ & $\begin{array}{c}p- \\
\text { value }\end{array}$ \\
\hline \multicolumn{5}{|c|}{ After 24 hours } \\
\hline Pain & $54(57.4 \%)$ & $49(52.1 \%)$ & $46(48.9 \%)$ & \multirow[b]{2}{*}{0.40} \\
\hline No Pain & $40(42.5 \%)$ & $45(47.8 \%)$ & $48(51 \%)$ & \\
\hline \multicolumn{5}{|c|}{ After 48 hours } \\
\hline Pain & $44(46.8 \%)$ & $42(44.6 \%)$ & $40(42.5 \%)$ & \multirow[b]{2}{*}{0.84} \\
\hline No Pain & $50(53.1 \%)$ & $52(55.3 \%)$ & $54(57.4 \%)$ & \\
\hline \multicolumn{5}{|c|}{ After 1 week } \\
\hline Pain & $26(27.6 \%)$ & $27(28.7 \%)$ & $20(21.2 \%)$ & \multirow[b]{2}{*}{0.45} \\
\hline No Pain & $68(72.3 \%)$ & $67(71.2 \%)$ & $74(78.7 \%)$ & \\
\hline
\end{tabular}

A study on the incidence of postoperative pain in teeth with apical periodontitis by Menakaya et al,11 concluded that calcium hydroxide and normal Saline combination was more effective in reducing pain than calcium hydroxide mixed with $0.2 \%$ chlorhexidine but the results were not statistically significant.

This coincides with our study that showed that although the results were not statistically significant, normal saline group was more effective in pain reduction than the chlorhexidine group. Another study by Ghanbarzadegan et al, ${ }^{12}$ conducted on different solution mixtures of Calcium Hydroxide for pain reduction coincided with our results and concluded that the 
results were not statistically significant and normal Saline, Chlorhexidine And Dexamethasone in combination with Calcium Hydroxide were all effective in pain reduction after endodontic therapy with Dexamethasone group showing the most pain reduction.

Corticosteroids have been used in dentistry either topically, systemically and intra-lesionally. They have been shown to effectively reduce pain after endodontic therapy. Patients mostly experience pain during the initial 24-72 hours period. ${ }^{11}$ Results of a systemic review concluded that the major effects of steroids on pain reduction occur during 24-72 hours and this coincides with our study. ${ }^{4}$

Another study by Mohammed Saadi Alarbeed et $a l,{ }^{14}$ comparing use of Calcium Hydroxide alone and in combination with chlorhexidine concluded that the mixture was more effective in reducing pain in necrotic teeth. The use of chlorhexidine as a potential intracanal medicament has proven to be effective because of its broad antimicrobial spectrum including enterococcus faecalis, and its ability to maintain its antibacterial action for a prolonged period of almost three months. ${ }^{15}$ A study conducted to compare the efficacy of different intra-canal medicaments found chlorhexidine to be more effective than Calcium Hydroxide in inhibiting growth of E. faecalis and post-operative pain. Thus, combining these two medicaments may result in the potentiation of their individual properties. ${ }^{16}$ According to Bernardi et al. ${ }^{17}$ Chlorhexidine has antimicrobial properties which are useful in endodontic treatment. A study by Khattak et al, ${ }^{18}$ comparing combination of Calcium Hydroxide with Chlorhexidine and normal Saline concluded that the former showed less mean pain score compared with the later.

There were a few limitations in the study conducted. A major drawback includes the absence of a control group against which all the three groups could be compared. It has been shown that endodontic therapy alone can result in pain reduction in patient presenting with pre-operative pain. ${ }^{19}$ In one study, endodontic therapy using placebo for managing pain showed that after six hours, $32.4 \%$ patients had no pain and after eight hours $41.2 \%$ patients had no pain. Similarly, in our study all the three groups showed decreased pain levels, making the patients believe that the medications reduced pain but the pain reduction may have been due to endodontic therapy alone. ${ }^{20,21}$

\section{CONCLUSION}

Calcium Hydroxide in combination with saline, chlorhexidine and dexamethasone are all effective for post- operative pain reduction during endodontic treatment with dexamethasone in combination with calcium hydroxide being the most effective out of the three combinations.

\section{Conflict of Interest: None.}

\section{Authors' Contribution}

AY: Supervisor the study, reviewed and did proof reading of the article, FA: Conceived the idea, planned the study and helped in manuscript writing, $\mathrm{AB}$ : Helped in manuscript writing and data collection, AMA: Substantial contribution to write up, literature review of the article and reference citation, SA: Helped in data collection.

\section{REFERENCES}

1. Valverde $M$, Baca $P$, Ceballos L, Fuentes $M$, Ruiz-Linares $M$, Ferrer-Luque C. Antibacterial efficacy of several intracanal medicaments for endodontic therapy. Dent Mater J 2017; 36(3): 319324.

2. Lakhani A, sekhar KS, Gupta P, Tejolatha B , Gupta A, Kashyap $\mathrm{S}$, et al. Efficacy of triple antibiotic paste, moxifloxacin, Calcium Hydroxide and 2\% Chlorhexidine gel in elimination of E. Faecalis: An in vitro study. J Clin Diagn Res 2017; 11(1): 6-9.

3. Athanassiadis B, Walsh LJ. Aspects of solvent chemistry for Calcium Hydroxide medicaments. Materials (Basel) 2017; 10(10): 1219.

4. Jafarzadeh H, Mohammadi Z, Shalavi S, Sahebalam R, Kinoshita J. Additive and reducing effects between Calcium Hydroxide and current irrigation solutions. J Contemp Dent Pract 2017; 18(3): 246-249.

5. Riaz A, Abdullah S, Saba K, Din SU. Comparison of two intracanal medicaments in resolution of apical radiolucency. J Ayub Med Coll 2018; 30(3): 320-324.

6. Midhat H, Hussein AG. Influence of calcium hydroxide chlorhexidine combination vs. calcium hydroxide as intra canal medicaments on postoperative flare-up following two-visit endodontic retreatment cases: single blinded randomized clinical trial. Acta Sci Dent Sci 2019; 10(3): 117-125.

7. Ranmanesh F, Parirokh M, Haghdoost AA, Abbott PV. Effect of corticosteroids on pain relief following root canal treatment: A Systematic Review. Iran Endod J 2017; 12(2): 123-130.

8. Manfredi M, Figini L, Gagliani M, Lodi G. Single versus multiple visits for endodontic treatment of permanent teeth. Cochrane Database Syst Rev 2016; 12(12): CD005296.

9. Ehrmann E, Messer H, Adams G. The relationship of intracanal medicaments to postoperative pain in endodontics. Int Endod J 2003; 36(12): 868-875.

10. Asnaashari M, Daghayeghi A, Mojahedi S, Azari-Marhabi S. Management of post endodontic retreatment pain with low level laser therapy. J Lasers Med Sci 2017; 8(3): 128-131.

11. Menakaya IN, Adegbulugbe IC, Oderinu OH, Shaba OP. The efficacy of Calcium Hydroxide powder mixed with $0.2 \%$ chlorhexidine digluconate or mixed with normal saline as intracanal medicament in the treatment of apical periodontitis. J Contemp Dent Pract 2015; 16(8): 657-664.

12. Ghanbarzadegan A, Ajami M, Aminsobhani M. The effect of different combinations of Calcium Hydroxide as intra-canal medicament on endodontic pain: A randomized clinical trial study. Iran Endod J 2019; 14(1): 1-6.

13. Farzaneh S, Parirokh M, Nakhaee N, Abbott PV. Effect of two different concentrations of sodium hypochlorite on postoperative pain following single-visit root canal treatment: a triple-blind randomized clinical trial. Int Endod J 2018; 51(Suppl-1): S2-S11. 


\section{Calcium Hydroxide}

14. Alarbeed MS, Ahmed GM. Effect of combination of calcium hydroxide and chlorhexidine gel $2 \%$ as intracanal medication in comparison to calcium hydroxide paste as intracanal medication on postoperative pain and bacterial endotoxin in necrotic teeth: a randomized controlled trial. Acta Sci Dent Sci 2019; 3(2): 124-130.

15. Miçooğulları K, Çalışkan, M. Efficacy of chlorhexidine as a final irrigant in one-visit root canal treatment: a prospective comparative study. Int Endod J 2018; 51(10): 1069-1076.

16. Lakhani AA, Sekhar KS, Gupta P, Tejolatha B, Gupta A, Kashyap $S$, et al. Efficacy of triple antibiotic paste, moxifloxacin, Calcium Hydroxide and 2\% chlorhexidine gel in elimination of e-faecalis: An in vitro study. J Clin Diagn Res 2017; 11(1): 6-9.

17. Bernardi A, Teixeira CS. The properties of chlorhexidine and undesired effects of its use in endodontics. Quintessence Int 2015; 46(7): 575-582.
18. Khattak YK, Shah SA, Alam F. Comparison of inter appointment pain between calcium hydroxide mixed with $2 \%$ Chlorhexidine and Calcium Hydroxide mixed with normal saline; a randomized controlled trial. J Khyber Coll Dent 2014; 5(1): 33-37.

19. Virdee S. Effective pain management strategies in endodontic therapy. Dent Update 2016; 43(6): 575-587.

20. Elzaki W, Abubakr N, Ziada H, Ibrahim Y. Double-blind randomized placebo-controlled clinical trial of efficiency of nonsteroidal anti-inflammatory drugs in the control of post-endodontic Pain. J Endod 2016; 42(6): 835-842.

21. Lin $\mathrm{C}, \mathrm{Wu} \mathrm{S}$, Yi C. association between anxiety and pain in dental treatment: A systematic review and meta-analysis. J Dent Res 2016; 96(2): 153-162. 